\title{
Asociación de variantes en genes de las proteínas desacoplantes con diabetes mellitus tipo 2 en una población del nordeste colombiano
}

\author{
Liliana Franco-Hincapié1, Constanza Elena Duque ${ }^{1}$, María Victoria Parra1', Natalia Gallego', \\ Alberto Villegas ${ }^{2}$, Andrés Ruiz-Linares ${ }^{1,3}$, Gabriel Bedoya ${ }^{1}$ \\ 1 Grupo de Genética Molecular, Universidad de Antioquia, Medellín, Colombia. \\ 2 Grupo de Endocrinología y Metabolismo, Hospital Universitario San Vicente de Paúl, \\ Universidad de Antioquia, Medellín, Colombia. \\ 3 The Galton Laboratory, University College London, London, United Kingdom.
}

Introducción. Las proteínas desacoplantes pertenecen a la familia de proteínas transportadoras de aniones que desacoplan la producción de ATP de la respiración mitocondrial, causando pérdida de protones a través de la membrana mitocondrial interna y disipando la energía en forma de calor. Aunque su función no ha sido bien establecida, algunos polimorfismos en estas proteínas se han asociado con diabetes mellitus tipo 2 , obesidad y resistencia a la insulina.

Objetivo. Evaluar la asociación entre las variantes -3826A/G, ID 45, -2723T/A, -1957G/A, -866G/A, $-55 \mathrm{C} / \mathrm{T}$ de los genes de las proteínas desacoplantes 1, 2 y 3 con diabetes mellitus tipo 2 en una población del nordeste colombiano.

Materiales y métodos. Se tipificaron 545 casos y 449 controles para 14 variantes de los genes de las proteínas desacoplantes por medio de PCR y PCR-RFLP. Se hicieron pruebas de asociación simples con ji al cuadrado y se corrigieron en un análisis de regresión logística bayesiana, incluyendo los estimados de mezcla individual obtenidos mediante 54 marcadores informativos de ascendencia europea, africana y amerindia.

Resultados. Las variantes $-3826 \mathrm{~A}(\mathrm{OR}=0,78$; IC95\% 0,63-0,97; $p=0,02),-55 \mathrm{C}(\mathrm{OR}=1,41$; IC95\% 1,04-1,92; $p=0,03)$ de las proteínas desacoplantes 1 y 3, respectivamente, y el haplotipo D45, -866G, -1957G, -2723T, -55C (OR=1,26; IC95\% 1,02-1,56; $p=0,03$ ) se asociaron con diabetes tipo 2. Estas asociaciones se conservaron después de ajustar por la mezcla genética individual. Conclusión. Algunas variantes de las proteínas desacoplantes 1, 2 y 3, y sus haplotipos, confieren riesgo para diabetes mellitus tipo 2 en una población del nordeste colombiano.

Palabras clave: diabetes mellitus/genética, resistencia a insulina, obesidad, genotipo, haplotipos.

Association between polymorphism in uncoupling proteins and type 2 diabetes in a northwestern Colombian population

Introduction. The uncoupling proteins belong to the family of anion transporting proteins which uncouple the ATP production from the mitochondrial respiration, cause proton leakage through the inner mitochondrial membrane, and release energy as heat. Although uncoupling protein function has not been well established, specific polymorphisms in these proteins have been associated with type 2 diabetes mellitus, obesity and insulin resistance.

Objective. The association was assessed between the polymorphisms in uncoupling protein genes 1, 2 and 3 genes and type 2 diabetes mellitus.

Materials and methods. In a northwestern Colombian population, 545 diabetes cases and 449 controls were investigated for presence of 14 polymorphisms in uncoupling protein genes (3826A/G, ID 45, 2723T/A, 1957G/A, 866G/A, and 55C/T) by PCR and PCR-RFLP. Single associations were evaluated by chi-square test, and bayesian logistic regression analysis was done including as covariates the individual admixture estimates obtained by 54 informative markers for European, African and Amerind ancestry.

Results. Association between type 2 diabetes mellitus and the polymorphisms 3826A (OR=0.78; $95 \% \mathrm{Cl}=0.63-0.97 ; p=0.02)$ and $55 \mathrm{C}(\mathrm{OR}=1.41 ; 95 \% \mathrm{Cl}=1.04-1.92 ; p=0.03)$ and the haplotype 
D45, 866G, 1957G, 2723T, and 55C (OR=1.26; 95\%Cl=1.02-1.56; $p=0.03)$ were found. These associations remained after adjustment using individual genetic admixture estimates.

Conclusion. Some alleles of uncoupling protein genes 1, 2 and 3, and their haplotypes confer risk to type 2 diabetes in a northwestern Colombian population.

Key words: Diabetes mellitus/genetics, insulin resistance, obesity, polymorphism, genotype, haplotypes.

De acuerdo con la edad de inicio, la dependencia a la insulina y otras características, la diabetes mellitus se clasifica en varios tipos, de los cuales, la diabetes mellitus tipo 2 representa el $90 \%$ de los casos diagnosticados; su incidencia en el mundo moderno ha alcanzado proporciones epidémicas, pues se ha estimado que actualmente afecta a más de 171 millones de personas y se predice que afectará a 366 millones para el año 2025 (1).

La diabetes mellitus tipo 2 está fuertemente asociada a la obesidad y su fisiopatología incluye dos defectos: la resistencia a la insulina en células de músculo esquelético, adipocito e hígado, y la compensación inadecuada de dicha resistencia por parte de las células beta del páncreas, para mantener la concentración de glucosa en sangre dentro del rango fisiológico normal (4 a $6 \mathrm{mmol} / \mathrm{L})$ (2). En la mayoría de los pacientes, la diabetes mellitus tipo 2 es el resultado de alteraciones en varios genes, cada uno con un efecto parcial y aditivo; estos genes pueden estar involucrados en la acción y la secreción de insulina, el desarrollo y la función de las células beta, el metabolismo y la obesidad. Además, los factores ambientales, como el sedentarismo y las dietas con alto contenido calórico, tienen un papel importante al favorecer el desarrollo de la enfermedad (3).

Los genes de las proteínas desacoplantes pertenecen a la familia de genes de las proteínas transportadoras de aniones; éstas pueden desacoplar la producción de ATP de la respiración mitocondrial y causar la pérdida de protones (4). Los genes de las proteínas desacoplantes

\footnotetext{
Correspondencia:

Liliana Franco-Hincapié, Sede de Investigación Universitaria, Universidad de Antioquia, Calle 62 No. 52-59, torre 2, laboratorio 430, Medellín, Colombia

Teléfono: (574) 219 6467; telefax: (574) 2196469

lifranco@udea.edu.co

Recibido: 11/04/08; aceptado:19/11/08
}

se han asociado con varias características fenotípicas implicadas en la diabetes mellitus tipo 2, como son la secreción de la insulina, la protección contra especies reactivas de oxígeno, la alteración en el metabolismo de los lípidos y la obesidad (5-7). Existe una gran homología entre sus secuencias: UCP2 y UCP3 tienen identidad con $U C P 1$ de $55 \%$ y $57 \%$, respectivamente; y UCP2 y UCP3 son $57 \%$ idénticas entre sí; el gen UCP1 está ubicado en la región 4q28-q31 y, UCP2 y UCP3, en la 11q13, con una distancia de $7 \mathrm{~kb}$ entre ellos (8).

Los genes de las proteínas desacoplantes tienen una expresión diferencial: UCP1 se expresa principalmente en el tejido adiposo pardo, la proteína UCP2 es ubicua y la UCP3 se encuentra en mayor cantidad en el tejido adiposo blanco y en el músculo esquelético; sin embargo, recientes investigaciones han mostrado la presencia de UCP1 y UCP3 en células beta pancreáticas $(5,9)$. Su función desacoplante se ha implicado con fenotipos asociados con diabetes mellitus tipo 2 en modelos animales y humanos. Estas relaciones varían según la proteína particular y su expresión en diferentes tejidos. En diversos estudios se han hallado, principalmente, relaciones entre alteraciones en UCP1 y UCP3, con obesidad y metabolismo de lípidos, en UCP2, con fallas en la secreción de insulina, y en UCP2 y UCP3, con protección contra especies reactivas de oxígeno $(6,7,10,11)$. Además, en el último año, UCP1 y UCP3 se han asociado con la secreción de insulina estimulada por glucosa $(5,9)$. Estos hallazgos son apoyados por la asociación de variantes en estos genes con las características fenotípicas mencionadas (11-13).

La combinación de ciertas características genéticas y determinados factores ambientales se ha asociado con la hipótesis de genotipo económico, propuesta por James Neel en 1962, la cual plantea que existe un conjunto de 
variantes genéticas que otorgan susceptibilidad a almacenar energía que, en condiciones de escasez, son una ventaja adaptativa $y$, en condiciones de abundancia, se expresa en forma de enfermedad, en este caso particular como diabetes mellitus tipo 2 (14).

Es probable que la población antioqueña posea un genotipo económico. Se ha demostrado que la prevalencia de diabetes mellitus tipo 2 está muy correlacionada con la ascendencia amerindia (15), que en poblaciones hispánicas de Estados Unidos las personas con ascendencia africana tiene mayor prevalencia de diabetes mellitus tipo 2 que las que poseen ascendencia europea (16) y que el riesgo para diabetes es significativamente menor en individuos con alta porcentaje de mezcla genética europea en comparación con la mezcla amerindia $(15,17)$. Los datos obtenidos a partir de marcadores clásicos (grupos sanguíneos y proteínas) sugieren que el componente genético de esta población es principalmente europeo $(70 \%)$, con una menor contribución amerindia y africana (15\% cada una) (18), y los porcentajes de los linajes del cromosoma Y (94\% europeo, 5\% africano y $1 \%$ amerindio) y del ADN mitocondrial (90\% amerindio, $8 \%$ africano y $2 \%$ europeo) muestran un sesgo en el patrón de cruces en la población fundadora (19).

Presentamos la hipótesis de que las variantes que confieren riesgo de desarrollar diabetes mellitus tipo 2 en la población antioqueña son heredadas de la población nativa de América y de la afroamericana, las cuales pueden estar comportándose como un genotipo económico. Esto pudo haber sucedido así si se tiene en cuenta que las poblaciones nativas de América debieron pasar por periodos de escasez de alimentos durante el viaje de Asia a este continente, que la historia de las poblaciones afroamericanas indica que pasaron por periodos de hambrunas y que, en este momento, muchas de ellas han cambiado su estilo de vida, en el cual el sedentarismo y la disponibilidad de alimentos con alto contenido energético se acompañan de un aumento de la prevalencia de diabetes mellitus tipo 2 (20).

Con base en esta teoría, la mezcla individual podría confundir los resultados de asociación entre polimorfismos de genes candidatos y diabetes mellitus tipo 2 , en loci donde las frecuencias difieran entre europeos, africanos y amerindios. Para contrarrestar esto, existe un modelo estadístico que permite ajustar las pruebas de asociación alélica y haplotípica para la mezcla individual como factor de confusión $(21,22)$.

De acuerdo con lo anterior, se quiso evaluar la asociación de variantes en los genes UCP con diabetes mellitus tipo $2 y$, por medio de marcadores informativos de ascendencia, controlar la mezcla genética individual como factor de confusión de la asociación.

\section{Materiales y métodos}

\section{Muestras}

Previo consentimiento informado, se tomaron muestras de sangre periférica de 545 individuos no relacionados (190 hombres/355 mujeres), con diagnóstico de diabetes mellitus tipo 2 (casos) según los criterios clínicos descritos por el comité experto en el diagnóstico y la clasificación de diabetes mellitus (23), y de 449 individuos asintomáticos (126 hombres/323 mujeres), no relacionados entre sí, mayores de 40 años, con un índice de masa corporal (IMC) menor de 30 y sin antecedentes personales o familiares de diabetes mellitus tipo 2 en primer grado, según la información suministrada por cada individuo (controles) (cuadro 1).

Los individuos que participaron en el estudio fueron contactados a través de diferentes instituciones, como el Hospital Universitario San Vicente de Paúl, el Seguro Social de Antioquia, el Laboratorio Clínico de la Universidad de Antioquia, Comfama y Susalud. A todos los participantes se les hizo una entrevista genealógica, previamente utilizada, que registra el lugar de nacimiento de los antecesores hasta los bisabuelos con el fin de establecer su procedencia $(19,24)$.

\section{Genotipificación}

A cada participante se le tomó una muestra de $10 \mathrm{ml}$ de sangre periférica, de la cual se extrajo ADN por el método estándar de fenolcloroformo. Para el análisis de asociación se 
evaluaron 14 polimorfismos, seis se encuentran en el gen UCP1, cuatro en UCP2 y cuatro en UCP3. De estos marcadores, 13 son cambios de un solo nucleótido y su genotipo se estableció por el método de reacción en cadena de la polimerasa-polimorfismos de longitud de los fragmentos de restricción (PCR-RFLP) y uno es una inserción/deleción de 45 pares de bases localizada en la región 3'UTR (exón 8) del gen UCP2 y su tipificación se basó en la diferencia del tamaño alélico. Los iniciadores y las enzimas de restricción utilizadas se encuentran en el anexo 1. Todos los productos de amplificación y los de digestión se resolvieron por medio de electroforesis en gel de agarosa al $3 \%$ teñida con bromuro de etidio.

Además, se utilizaron 54 marcadores informativos de ascendencia (Ancestry Informative Marker) cuyo genotipo se estableció previamente; estos marcadores se caracterizan por tener una gran diferencia en las frecuencias alélicas entre poblaciones, por lo que permiten evaluar el grado de mezcla genética (25) (anexo 2).

\section{Análisis estadístico}

Se utilizó el programa GENEPOP 3.1 (26) para calcular las frecuencias alélicas y las genotípicas, para hacer las pruebas de equilibrio de HardyWeinberg y de estructuración de población; el programa ARLEQUIN 2000 (27) se utilizó para generar los loci compuestos (haplotipos más probables) y calcular el patrón de desequilibrio de ligamiento (D'); se utilizó GraphPad InStat 3 (http://www.graphpad.com/instat/instat.htm) para realizar las pruebas de ji al cuadrado $\left(\chi^{2}\right)$ y calcular las razones de disparidad y, ADMIXMAP $3.8(21,28)$, para estimar la proporción de mezcla

Cuadro 1. Características de la población de estudio*.

\begin{tabular}{lccc}
\hline & $\begin{array}{c}\text { Pacientes } \\
\text { diabéticos }\end{array}$ & $\begin{array}{c}\text { Controles no } \\
\text { diabéticos }\end{array}$ & $\mathrm{p}$ \\
\hline Número & 545 & 449 & - \\
Edad (años) & $58,0 \pm 10,7$ & $60,6 \pm 10,2$ & $<0,001$ \\
Sexo (M/F) & $190 / 355$ & $126 / 323$ & 0,024 \\
Edad de diagnóstico & $59,9 \pm 11,5$ & - & - \\
(años) $^{*}$ & & & \\
IMC $\left(\mathrm{kg} / \mathrm{m}^{2}\right)^{*}$ & $27,0 \pm 4,6$ & $25,2 \pm 3,8$ & $<0,001$ \\
\hline
\end{tabular}

*Los datos están representados por la media \pm desviación estándar individual y la corrección con mezcla genética de la asociación alélica y haplotípica de los marcadores polimórficos en los genes UCP.

\section{Consideraciones éticas}

Se tuvieron en cuenta las consideraciones éticas para los estudios de investigación en salud, para la aprobación por el Comité de Ética de la Corporación para el Estudio de Patologías Tropicales adscrita a la Universidad de Antioquia. Los pacientes fueron informados de los riesgos y beneficios que implicaban su participación en esta investigación, previa firma del conocimiento informado.

\section{Resultados \\ Grado de mezcla genética}

No se hallaron indicios de estructuración de población medidos mediante el estadístico Fst a partir de los 54 marcadores informativos de ascendencia $(0,0091)$.

Los pacientes con diabetes mellitus tipo 2 presentaron un promedio de porcentaje de mezcla individual de $57,8 \pm 0,12 \%$ europea, $11,2 \pm 0,08 \%$ africana y $30,9 \pm 0,09 \%$ amerindia y, los controles, de $59,6 \pm 0,11 \%$ europea, $9,4 \pm 0,07 \%$ africana y $30,9 \pm 0,08 \%$ amerindia.

Aunque estas diferencias no fueron significativas para el porcentaje de mezcla amerindia, el cual fue igual entre casos y controles $(p=0,668)$, se encontró un mayor porcentaje de mezcla europea en los controles $(p=0,001)$ y un mayor porcentaje de mezcla africana en los casos $(p=0,001)$. Estos datos pueden indicar que la ascendencia europea ejerce un efecto protector para diabetes mellitus tipo 2 y que el genotipo económico que está asociado con diabetes mellitus tipo 2 en esta población puede provenir del grado de mezcla africana. Este planteamiento merece investigaciones adicionales en el futuro.

\section{Variantes de los genes de las proteínas desacoplantes y su asociación con diabetes tipo 2}

De los seis marcadores cuyo genotipo se estableció para el gen UCP1, 5 son monomórficos, con los alelos Arg40, Ala64, Val137, Met229 y Lys257 fijos en la población antioqueña; los alelos 
Val202, Arg143 y IVS5+1G de los marcadores tipificados para el gen UCP3, también se encuentran fijos en esta población.

Todos los polimorfismos evaluados de los genes UCP se encuentran en equilibrio de HardyWeinberg para casos y controles; además, no se encontraron indicios de estructuración de población medida a partir del estadístico Fst con estos marcadores (no se presentan los resultados).

Se encontraron diferencias significativas en las distribución de las frecuencias alélicas entre los casos y los controles para los marcadores -3826A/G de UCP1 (OR=0,78; IC95\% 0,63$0,97 ; p=0,02)$ y $-55 \mathrm{C} / \mathrm{T}$ de UCP3 $(\mathrm{OR}=1,41$; IC $95 \% 1,04-1,92 ; p=0,03$ ) evaluadas por medio de pruebas de $\chi^{2}$ (cuadro 2); para verificar que la asociación no sea consecuencia de las diferencias en el porcentaje de mezcla genética de los casos y de los controles y, por lo tanto, sean asociadas al estatus de salud o enfermedad, se realizó un ajuste teniendo en cuenta la ascendencia genética individual como factor de confusión; ambas asociaciones se conservaron después de este ajuste (cuadro 2). De acuerdo con lo anterior, el alelo -3826A del gen UCP1 presenta asociación negativa con diabetes mellitus tipo 2 con una razón de disparidad (OR) de 0,79 $(p=0,04)$, por lo que confiere protección para diabetes mellitus tipo 2 , y el alelo $-55 \mathrm{C}$ de UCP3 confiere riesgo para desarrollar diabetes mellitus tipo 2 en la población estudiada con un OR de $1,38(p=0,04)$.

No se encontraron diferencias significativas, entre los casos y los controles, para las frecuencias genotípicas de ninguno de los marcadores (cuadro 3).

\section{Locus compuesto}

El patrón de desequilibrio de ligamiento por pares difiere entre casos y controles; en general, se presentaron valores D' mayores en el grupo de casos (cuadro 4). Todos los valores D' entre el polimorfismo -3826A/G de UCP1 y los polimorfismos de los otros dos genes, son menores de 0,5 , lo que es muy coherente teniendo en cuenta que se encuentra en un cromosoma diferente. Por lo tanto, éste no se tuvo en cuenta para el análisis de haplotipos. De los 32 haplotipos probables, en los casos se obtuvieron 20 y en los controles 26; por lo tanto, estos últimos tienen mayor diversidad haplotípica.

Se encontraron siete haplotipos con una frecuencia mayor de 0,1 , presentes en ambos grupos (cuadro 5); los haplotipos raros se agruparon en una sola categoría y a todos los haplotipos se les hizo una prueba de asociación con diabetes mellitus tipo 2. El haplotipo modal DGGTC tiene una frecuencia de 0,48 en casos y 0,42 en controles, por lo que está asociado de manera significativa a diabetes mellitus tipo

Cuadro 2. Frecuencias alélicas en los casos y los controles, y pruebas de asociación entre diabetes mellitus tipo 2 y las variantes en los genes que codifican para las proteínas desacoplantes.

\begin{tabular}{|c|c|c|c|c|c|c|c|}
\hline \multirow[t]{2}{*}{ Gen } & \multirow[t]{2}{*}{ Alelo } & \multirow[t]{2}{*}{$\begin{array}{c}\text { Frecuencia } \\
\text { en casos }\end{array}$} & \multirow[t]{2}{*}{$\begin{array}{l}\text { Frecuencia } \\
\text { en controles }\end{array}$} & \multicolumn{2}{|c|}{ Prueba de $\chi^{2}$} & \multicolumn{2}{|c|}{$\begin{array}{l}\text { Regresión bayesiana } \\
\text { ajustada por mezcla } \\
\text { genética individual }\end{array}$} \\
\hline & & & & Valor $p$ & OR (IC95\%) & Valor $p$ & $\mathrm{OR}^{\star *}$ \\
\hline UCP1 & $-3826 A$ & 0,54 & 0,60 & 0,02 & $0,78(0,63-0,97)$ & 0,04 & 0,79 \\
\hline UCP2 & $\begin{array}{l}D 45 \\
-2723 T \\
-1957 G \\
-866 \mathrm{G}\end{array}$ & $\begin{array}{l}0,73 \\
0,61 \\
0,81 \\
0,62\end{array}$ & $\begin{array}{l}0,69 \\
0,56 \\
0,81 \\
0,58\end{array}$ & $\begin{array}{l}0,10 \\
0,07 \\
1,00 \\
0,07\end{array}$ & $\begin{array}{c}1,22(0,97-1,54) \\
1,20(0,99-1,52) \\
1,00(0,76-1,3) \\
1,23(0,99-1,92)\end{array}$ & $\begin{array}{l}0,12 \\
0,10 \\
0,62 \\
0,17\end{array}$ & $\begin{array}{l}1,21 \\
1,20 \\
0,93 \\
1,17\end{array}$ \\
\hline UCP3 & $-55 C$ & 0,88 & 0,84 & 0,03 & $1,41(1,04-1,92)$ & 0,04 & 1,38 \\
\hline
\end{tabular}

${ }^{*}$ Resultados según las covariables edad y sexo.

${ }^{* *} \mathrm{OR}=\mathrm{e}^{\text {(score/porcentaje de información observada) }}$. Estos resultados se obtienen del programa ADMIXMAP 3.8. 
$2(\mathrm{OR}=1,26 ; \operatorname{IC} 95 \%$ 1,02-1,56; $\mathrm{p}=0,03)$. Esta asociación permanece después de la corrección por mezcla genética y muestra un OR de 1,31 $(p=0,01)$ (cuadro 5).

\section{Genotipos multiloci}

Se realizó una prueba de asociación entre los genotipos multiloci compuestos por los dos polimorfismos asociados de manera individual con diabetes mellitus tipo $2(-3826 \mathrm{~A} / \mathrm{G}$ de UCP1 y $-55 \mathrm{C} / \mathrm{T}$ de UCP3) (cuadro 6). El genotipo $-3826 \mathrm{~A} / \mathrm{A}, \quad-55 \mathrm{C} / \mathrm{T}$ se encontró asociado de manera significativa con menor riesgo de sufrir la enfermedad (OR=0,39; IC95\% 0,21-0,71; $\mathrm{p}=0,002)$.

\section{Discusión}

En este estudio se encontró una asociación entre los polimorfismos $-3826 \mathrm{~A} / \mathrm{G}$ de $U C P 1,-55 \mathrm{C} / \mathrm{T}$ de UCP3 y el haplotipo DGGTC con diabetes tipo 2, controlando el efecto de la mezcla como factor de confusión. Los estimativos de mezcla genética europea y africana son significativamente diferentes entre casos y controles; la primera fue mayor en los controles y, la segunda, mayor en los casos. Después de hacer un ajuste

Cuadro 3. Frecuencias genotípicas en los casos y los controles.

\begin{tabular}{|c|c|c|c|c|c|c|c|c|c|}
\hline \multirow{3}{*}{$\frac{\text { Gen }}{\text { UCP1 }}$} & \multirow{2}{*}{$\begin{array}{c}\text { Polimorfismo* } \\
-3826 \mathrm{~A} / \mathrm{G}\end{array}$} & \multirow{2}{*}{$\begin{array}{l}\text { Valor } \mathbf{p}^{\text {** }} \\
0,06\end{array}$} & \multirow{2}{*}{$\begin{array}{c}\text { OR }(\text { IC95\%) })^{\star * *} \\
-/ G\end{array}$} & \multicolumn{3}{|c|}{ Casos } & \multicolumn{3}{|c|}{ Controles } \\
\hline & & & & GG & $\mathbf{A G}$ & AA & GG & $\mathbf{A G}$ & AA \\
\hline & & & $1,36(0,99-1,9)$ & 0,22 & 0,49 & 0,30 & 0,16 & 0,47 & 0,36 \\
\hline \multirow[t]{7}{*}{ UCP2 } & ID 45 & 0,13 & $\begin{array}{c}\text { DD } \\
1,30(0,94-1,7)\end{array}$ & $\begin{array}{c}\text { II } \\
0,07\end{array}$ & $\begin{array}{l}\text { ID } \\
0,40\end{array}$ & $\begin{array}{c}\text { DD } \\
0,53\end{array}$ & $\begin{array}{c}\text { II } \\
0,09\end{array}$ & $\begin{array}{c}\text { ID } \\
0,44\end{array}$ & $\begin{array}{l}\text { DD } \\
0,47\end{array}$ \\
\hline & $-2723 T / A$ & 0,19 & AA & AA & TA & AA & $\mathbf{A A}$ & TA & AA \\
\hline & & & $1,3(0,87-1,94)$ & 0,15 & 0,48 & 0,38 & 0,19 & 0,51 & 0,30 \\
\hline & $-1957 G / A$ & 0,90 & $-/ A$ & AA & GA & TT & AA & GA & TT \\
\hline & & & $1,01(0,94-1,70)$ & 0,03 & 0,31 & 0,65 & 0,03 & 0,31 & 0,65 \\
\hline & $-866 G / A$ & 0,07 & GG & AA & GA & GG & AA & GA & GG \\
\hline & & & $1,34(0,98-1,82)$ & 0,14 & 0,46 & 0,39 & 0,17 & 0,51 & 0,32 \\
\hline \multirow[t]{2}{*}{ UCP3 } & $-55 \mathrm{C} / \mathrm{T}$ & 0,06 & CC & $\mathrm{TT}$ & СТ & CC & TT & CT & CC \\
\hline & & & $1,41(1,00-1,90)$ & 0,02 & 0,20 & 0,78 & 0,03 & 0,25 & 0,71 \\
\hline
\end{tabular}

* El alelo resaltado en negrilla en cada polimorfismo es el más frecuente en los casos.

** Valor $\mathrm{p}$ de estimado a partir de la comparación entre las distribuciones de las frecuencias genotípicas entre casos y controles

*** El símbolo -/G hace referencia a los genotipos GG y AG de la variante -3826A/G de UCP1; y el símbolo -/A hace referencia a los genotipos A/A y -/A de la variante -866G/A de UCP2.

Cuadro 4. Desequilibrio de ligamiento en los casos y los controles ${ }^{\star *}$.

\begin{tabular}{|c|c|c|c|c|c|c|c|c|}
\hline \multirow[b]{2}{*}{ D' controles } & \multicolumn{8}{|c|}{ D' casos } \\
\hline & \multicolumn{3}{|c|}{ UCP1 } & \multicolumn{2}{|c|}{ UCP2 } & \multirow[b]{2}{*}{$-866 \mathrm{G} / \mathrm{A}$} & \multirow[b]{2}{*}{ I/D45 } & \multirow{2}{*}{$\begin{array}{l}\text { UCP3 } \\
-55 \mathrm{C} /\end{array}$} \\
\hline & * & & $-3826 A / G$ & $-2723 T / A$ & $-1957 G / A$ & & & \\
\hline & UCP1 & $-3826 A / G$ & * & 0,11 & 0,11 & 0,12 & 0,34 & 0,50 \\
\hline & UCP2 & $-2723 T / A$ & 0,33 & * & 0,54 & 0,94 & 0,88 & 0,44 \\
\hline & & $-1957 \mathrm{G} / \mathrm{A}$ & 0,24 & 0,61 & * & 0,57 & 0,44 & 0,58 \\
\hline & & $-866 \mathrm{G} / \mathrm{A}$ & 0,27 & 0,92 & 0,56 & * & 0,93 & 0,44 \\
\hline & & I/D45 & 0,26 & 0,85 & 0,60 & 0,85 & * & 0,95 \\
\hline & UCP3 & $-55 \mathrm{C} / \mathrm{T}$ & 0,01 & 0,35 & 0,54 & 0,32 & 0,92 & * \\
\hline
\end{tabular}

${ }^{* \star}$ Desequilibrio de ligamiento estimado como D' 
teniendo en cuenta la mezcla genética como factor de confusión, se conservó la asociación encontrada entre diabetes mellitus tipo 2 y los polimorfismos $-3826 \mathrm{~A} / \mathrm{G}$ de UCP1, $-55 \mathrm{C} / \mathrm{T}$ de UCP3 y el haplotipo DGGTC. Estos resultados indican que existe una relación certera entre estas variantes y la diabetes mellitus tipo 2 , y no una falsa interpretación de asociación debida a mezcla genética.

El alelo -3826A del gen UCP1 puede conferir un efecto protector para diabetes mellitus tipo 2 en la población estudiada. Estos resultados son coherentes con investigaciones previas, en las que el alelo -3826G se asoció con un menor efecto térmico como respuesta a una alimentación alta en grasa en niños con posibles efectos adversos en la regulación del peso corporal (29), mayor índice de masa corporal $(30,31)$ en adultos obesos, y mayor porcentaje de grasa corporal $(30,32)$ y obesidad $(13,33)$.

La UCP1 se expresa de manera simultánea con la insulina en los islotes pancreáticos de ratón, mono y humano; existe asociación del alelo -3826G con la varianza en la respuesta aguda de insulina inducida por glucosa (9) y este alelo disminuye la expresión de insulina en tejido adiposo (34). Además, los estudios de aumento de la expresión en células de insulinoma muestran que la regulación positiva de UCP1 suprime la secreción de insulina (35) y el desacoplamiento respiratorio mediado por UCP1 en tejido muscular esquelético tuvo un efecto inverso sobre la resistencia a la insulina en ratones obesos y transgénicos para UCP1,

Cuadro 5. Frecuencias haplotípicas en los casos y en los controles, y pruebas de asociación entre diabetes tipo 2 y los haplotipos más frecuentes conformados por las variantes de los genes de las proteínas desacoplantes 2 y 3 .

\begin{tabular}{ccccccc}
\hline $\begin{array}{c}\text { Haplotipo* } \\
\text { ID45, -866G/A, -1957G/A, -2723T/A, -55C/T }\end{array}$ & $\begin{array}{c}\text { Frecuencia } \\
\text { en casos }\end{array}$ & $\begin{array}{c}\text { Frecuencia } \\
\text { en controles }\end{array}$ & Pruebas $\chi^{2}$ & $\begin{array}{c}\text { Regresión bayesiana } \\
\text { ajustada por mezcla } \\
\text { genética individual }\end{array}$ \\
\hline IAGAC & & & Valor $p \quad$ OR (IC95\%) & Valor $p$ & OR ${ }^{\star *}$ \\
DAAAT & 0,24 & 0,26 & 0,36 & $0,89(0,70-1,13)$ & 0,33 & 0,88 \\
DAAAC & 0,08 & 0,09 & 0,59 & $0,87(0,59-1,28)$ & 0,45 & 0,86 \\
DGATC & 0,03 & 0,03 & 1,00 & $1,02(0,57-1,83)$ & 0,74 & 1,11 \\
DGGAC & 0,07 & 0,05 & 0,14 & $1,41(0,91-2,19$ & 0,17 & 1,43 \\
DGGTT & 0,03 & 0,02 & 0,73 & $1,17(0,59-2,32)$ & 0,90 & 0,95 \\
DGGTC & 0,03 & 0,04 & 0,47 & $0,77(0,43-1,38)$ & 0,35 & 0,74 \\
Otros & 0,48 & 0,42 & 0,03 & $1,26(1,02-1,56)$ & 0,01 & 1,31 \\
\hline
\end{tabular}

*Haplotipos con frecuencia mayor de 0,01

${ }^{* *} \mathrm{OR}=\mathrm{e}^{\text {(score/porcentaje de información observada) }}$. Estos resultados se obtienen del programa ADMIXMAP 3.8.

Cuadro 6. Genotipos multiloci conformados por las variantes asociadas con diabetes mellitus tipo 2.

\begin{tabular}{lcccc}
\hline $\begin{array}{l}\text { Genotipo multiloci } \\
\text {-3826A/G, -55C/T* }\end{array}$ & Valor $\mathbf{p}$ & OR (IC95\%) & Casos & Controles \\
\hline A/A, C/C & 0,86 & $1,03(0,73-1,46)$ & 0,25 & 0,24 \\
A/A, C/T & 0,002 & $0,39(0,21-0,71)$ & 0,05 & 0,11 \\
A/A, T/T & 0,14 & $0,14(0,01-2,71)$ & 0,00 & 0,01 \\
A/G, C/C & 0,88 & $1,03(0,73-1,40)$ & 0,37 & 0,37 \\
A/G, C/T & 0,53 & $1,19(0,73-1,97)$ & 0,11 & 0,09 \\
A/G, T/T & 0,48 & $0,44(0,09-2,18)$ & 0,006 & 0,01 \\
G/G, C/C & 0,06 & $1,56(1,01-2,41)$ & 0,16 & 0,11 \\
G/G, C/T & 1,0 & $1,02(0,50-2,10)$ & 0,04 & 0,04 \\
G/G, T/T & 0,73 & $1,32(0,33-5,34)$ & 0,01 & 0,01 \\
\hline
\end{tabular}

*Polimorfismos asociados a diabetes mellitus tipo 2 de manera individual. 
en comparación con los obesos no transgénicos (36). Todos estos estudios apuntan hacia una posible relación del polimorfismo de UCP1, no sólo con obesidad, sino también con resistencia a la insulina.

La expresión de UCP1 en cerebro, músculo esquelético, útero y timo de ratón (37-39), hace indispensable estudiar más exhaustivamente la presencia de su proteína en diferentes tejidos humanos, pues esto podría cambiar ampliamente el panorama y permitir encontrar explicaciones a la relación que parece evidente dada la concordancia en muchos estudios diferentes. Además, es importante tener en cuenta la relación de UCP1 con muchas otras moléculas que pueden alterar su expresión, como es el caso de los receptores activados del proliferador del peroxisoma alfa y gamma (PPAR $\alpha, \gamma)$, receptores de ácido retinoico (40), cuyas interacciones podrían dar explicaciones a los resultados encontrados.

La asociación entre el alelo -55C de UCP3 y diabetes mellitus tipo 2 encontrada en este análisis, está de acuerdo con estudios en los que se han descrito las asociaciones del alelo -55T con menor riesgo de desarrollar diabetes mellitus tipo 2 (41) y con bajo contenido de grasa en mujeres que consumen dietas ricas en calorías (42). El alelo -55C de UCP3 podría tener un papel funcional, dado que se ha asociado con una menor cantidad de ARNm y de proteína en músculo esquelético $(43,44)$.

Se ha encontrado una relación negativa entre la expresión de UCP3 y los niveles de glucosa e insulina en plasma $(11,45)$, resistencia a la insulina en ratones transgénicos sometidos a dietas altas en grasa (46) y el índice de masa corporal (43). Además, recientemente se demostró la presencia de UCP3 en islotes pancreáticos humanos y el papel de esta proteína en la homeostasis de la glucosa. Aunque su expresión es de sólo 10\% comparado con la de UCP2, se halló que una exposición crónica de los islotes a concentraciones altas en glucosa atenúan la expresión de UCP3, mientras que la UCP2 se incrementa y que expresión exagerada de UCP3 y la baja expresión de UCP2 resultan en un incremento de 1,4 veces la sensibilidad de insulina estimulada por glucosa en islotes aislados de humanos $(11,34)$.

El haplotipo modal D45, -866G, -1957G, -2723T, $-55 \mathrm{C}$ se encuentra asociado de manera significativa con la diabetes mellitus tipo 2. En ningún reporte revisado hasta la fecha, se ha estudiado de manera conjunta estos cinco polimorfismos que se encuentran en una región de menos de $35 \mathrm{~kb}$ en la posición 11q13, pero sí se han realizado estudios en los que se tienen en cuenta varios de ellos $(12,47,48)$.

Esterbauer y colaboradores hicieron un estudio de asociación entre los polimorfismos -2723T/A, -1957G/A, -866G/A, I/D13 e l/D45 del gen UCP2 y la obesidad, y encontraron resultados similares a los nuestros con asociaciones positivas para el marcador $-866 \mathrm{G} / \mathrm{A}$. En un análisis de haplotipos con tres polimorfismos, el locus compuesto -1957G, -866G, D45 se encontraba con mayor frecuencia en individuos obesos comparados con delgados, sin diferencias significativas entre los grupos (47). En un estudio prospectivo, encontraron que el haplotipo -866A, -55T del gen UCP2 aumenta la probabilidad de sufrir diabetes mellitus tipo 2 después de 10 años, pero se pierde la significancia estadística para el análisis luego de 15 años (12). Por último, en una investigación reciente encontraron que el haplotipo -866G, D45, -55T, conformado por dos polimorfismos de UCP2 y uno de UCP3, respectivamente, se encuentra asociado a obesidad; sin embargo, esta asociación tampoco es significativa (48).

De acuerdo con lo anterior y teniendo en cuenta que el haplotipo que contiene los alelos D45, -2723T y -55C se encuentra con mayor frecuencia en casos que en controles y el alelo -1957G se encuentra con igual frecuencia en los dos grupos, es muy coherente que el locus compuesto DGGTC, se encuentre asociado a mayor riesgo de sufrir diabetes mellitus tipo 2 . El hecho de que esto no esté de acuerdo con algunos otros trabajos, puede ser el reflejo de las diferencias en las poblaciones estudiadas.

Este estudio presenta una debilidad en la selección de los controles, puesto que no se 
realizaron las pruebas de laboratorio estándar para confirmar el estatus de no diabético; sin embargo, sólo se incluyeron los individuos que negaron antecedentes personales y familiares de diabetes y se verificó la ausencia de obesidad en todos ellos. La probabilidad de haber incluido casos subclínicos de diabetes en el grupo control es baja, dado que los criterios de selección (entrevista y medición de obesidad) filtran un alto porcentaje de enfermos. Además, esta probabilidad disminuye si se tiene en cuenta que en la población general de Colombia la prevalencia de diabetes varía entre 5\% y 7\% $(49,50)$, y que la presencia de obesidad e historia familiar de diabetes tipo 2 está muy relacionada con la enfermedad (51-53).

La información recolectada para casos y controles no es completamente comparable; sin embargo, consideramos que la asociación significativa observada entre la exposición (genotipo) y la diabetes es sólida, dado que se presentó a pesar de este posible sesgo que, en el caso de que estuviera presente, conduciría a una mala clasificación de algunos de los controles y a desviar la asociación hacia el valor nulo (54).

A pesar de los múltiples estudios con resultados positivos de asociación entre diferentes variantes de los genes UCP y los fenotipos implicados con diabetes mellitus tipo 2, es importante la verificación de estos resultados con estudios clínicos prospectivos. Al respecto, en un estudio de cohortes de una población de hombres caucásicos, se analizó la asociación de diabetes mellitus tipo 2 y dos de las variantes incluidas en este estudio (-866G/A de UCP2 y $-55 \mathrm{C} / \mathrm{T}$ de UCP3), con resultados positivos (12); en el trabajo de Cheurfa y colaboradores se encontró que el alelo -866A de UCP2 tiene una relación inversa con el riesgo de desarrollar enfermedad arterial coronaria en personas caucásicas con diabetes mellitus tipo 2 (55). Estos resultados positivos en análisis prospectivos confirman la asociación de los UCP con diabetes mellitus tipo 2, al menos para algunas poblaciones. Es necesario, entonces, consolidar nuestros resultados mediante este tipo de estudios en nuestra población.

\section{Agradecimientos}

Este estudio fue posible gracias al apoyo institucional de la Universidad de Antioquia y del Hospital Universitario San Vicente de Paúl, Seguro Social de Antioquia, Susalud EPS, IPS universitaria, Laboratorio Clínico de la Escuela de Microbiología de la Universidad de Antioquia, cajas de compensación familiar COMFAMA y COMFENALCO y a todas las personas que amablemente colaboraron con la donación de sus muestras.

Agradecemos a David Reich, del Departamento de Genética de la Escuela de Medicina de la Universidad de Harvard, por suministrar los genotipos de los AIM y a Candelaria Vergara y Ana Victoria Valencia por sus valiosos aportes en la edición del manuscrito.

\section{Conflicto de intereses}

Los autores del presente manuscrito certificamos que no existe ningún tipo de conflicto de interés en su elaboración.

\section{Financiación}

Este estudio hace parte de dos proyectos de investigación financiados por Colciencias, códigos 11150416415 y 11150412986.

\section{Referencias}

1. Zimmet P, Alberti KG, Shaw J. Global and societal implications of the diabetes epidemic. Nature. 2001;414:782-7.

2. McCarthy MI. Progress in defining the molecular basis of type 2 diabetes mellitus through susceptibility-gene identification. Hum Mol Genet. 2004;13:33-41.

3. Tusie Luna MT. Genes and type 2 diabetes mellitus. Arch Med Res. 2005;36:210-22.

4. Sokolova IM, Sokolov EP. Evolution of mitochondrial uncoupling proteins: novel invertebrate UCP homologues suggest early evolutionary divergence of the UCP family. FEBS Lett. 2005;579:313-7.

5. Li Y, Maedler K, Shu L, Haataja L. UCP-2 and UCP-3 proteins are differentially regulated in pancreatic betacells. PLoS ONE. 2008;3:e1397.

6. Nedergaard J, Cannon B. The 'novel' 'uncoupling' proteins UCP2 and UCP3: what do they really do? Pros and cons for suggested functions. Exp Physiol. 2003;88:65-84.

7. Rousset S, Alves-Guerra MC, Mozo J, Miroux B, Cassard-Doulcier AM, Bouillaud F, et al. The biology 
of mitochondrial uncoupling proteins. Diabetes. 2004;53 (Suppl.1):S130-5.

8. Pawade T, Ho PW, Kwok KH, Chu AC, Ho SL, Ramsden DB. Uncoupling proteins: targets of endocrine disruptors? Mol Cell Endocrinol. 2005;244:79-86.

9. Sale MM, Hsu FC, Palmer ND, Gordon CJ, Keene $\mathrm{KL}$, Borgerink HM, et al. The uncoupling protein 1 gene, UCP1, is expressed in mammalian islet cells and associated with acute insulin response to glucose in African American families from the IRAS Family Study. BMC Endocr Disord. 2007;7:1.

10. Chan CB, Saleh MC, Koshkin V, Wheeler MB. Uncoupling protein 2 and islet function. Diabetes 2004;53 (Suppl.1):S136-42.

11. Hsu YH, Niu T, Song Y, Tinker L, Kuller LH, Liu S. Genetic variants in the UCP2-UCP3 gene cluster and risk of diabetes in the Women's Health Initiative Observational Study. Diabetes. 2008;57:1101-7.

12. Gable DR, Stephens JW, Cooper JA, Miller GJ, Humphries SE. Variation in the UCP2-UCP3 gene cluster predicts the development of type 2 diabetes in healthy middle-aged men. Diabetes. 2006;55:1504-11.

13. Shin HD, Kim KS, Cha MH, Yoon Y. The effects of UCP-1 polymorphisms on obesity phenotypes among Korean female subjects. Biochem Biophys Res Commun. 2005;335:624-30.

14. Neel JV. Diabetes mellitus: a "thrifty" genotype rendered detrimental by "progress"? Am J Hum Genet. 1962;14:353-62.

15. Chakraborty R, Ferrell RE, Stern MP, Haffner SM, Hazuda HP, Rosenthal M. Relationship of prevalence of non-insulin-dependent diabetes mellitus to Amerindian admixture in the Mexican Americans of San Antonio, Texas. Genet Epidemiol. 1986;3:435-54.

16. Harris MI, Flegal KM, Cowie CC, Eberhardt MS, Goldstein DE, Little RR, et al. Prevalence of diabetes, impaired fasting glucose, and impaired glucose tolerance in U.S. adults. The Third National Health and Nutrition Examination Survey, 1988-1994. Diabetes Care. 1998;21:518-24.

17. Williams RC, Long JC, Hanson RL, Sievers ML, Knowler WC. Individual estimates of European genetic admixture associated with lower body-mass index, plasma glucose, and prevalence of type 2 diabetes in Pima Indians. Am J Hum Genet. 2000;66:527-38.

18. Sandoval C, De la Hoz A, Yunis E. Estructura genética de la población colombiana. Revista Facultad de Medicina Universidad Nacional de Colombia. 1993;41:3-14.

19. Bedoya G, Montoya P, García J, Soto I, Bourgeois S, Carvajal L, et al. Admixture dynamics in Hispanics: a shift in the nuclear genetic ancestry of a South American population isolate. Proc Natl Acad Sci USA. 2006;103:7234-9.
20. Gower BA, Fernández JR, Beasley TM, Shriver MD, Goran MI. Using genetic admixture to explain racial differences in insulin-related phenotypes. Diabetes. 2003;52:1047-51.

21. Hoggart CJ, Parra EJ, Shriver MD, Bonilla C, Kittles RA, Clayton DG, et al. Control of confounding of genetic associations in stratified populations. Am J Hum Genet. 2003;72:1492-504.

22. Parra EJ, Hoggart CJ, Bonilla C, Dios S, Norris JM, Marshall JA, et al. Relation of type 2 diabetes to individual admixture and candidate gene polymorphisms in the Hispanic American population of San Luis Valley, Colorado. J Med Genet. 2004;41:e116.

23. The Expert Committee on the Diagnosis and Classification of Diabetes Mellitus. Report of the Expert Committee on the Diagnosis and Classification of Diabetes Mellitus Diabetes Care. 2002;25.

24. Bedoya G, García J, Montoya P, Rojas W, Amézquita ME, Soto I, et al. Análisis de isonimia entre poblaciones del noroeste de Colombia. Biomédica. 2006;26:538-45.

25. Shriver MD, Smith MW, Jin L, Marcini A, Akey JM, Deka $\mathbf{R}$, et al. Ethnic-affiliation estimation by use of population-specific DNA markers. Am J Hum Genet. 1997;60:957-64.

26. Raymond M, Rousset F. GENEPOP (version 1.2): population genetics software for exact tests and ecumenicism. J Hered. 1995;86.

27. Schneider S, Roessli D, Excoffier L. Arlequin: A software for population genetics data analysis. Versión 2000. Ginebra: Laboratorio de Genética y Biometría, Departamento de Antropología, Universidad de Ginebra; 2000.

28. McKeigue PM, Carpenter JR, Parra EJ, Shriver MD. Estimation of admixture and detection of linkage in admixed population s by a Bayesian approach: application to African-American populations. Ann Hum Genet. 2000;64:171-86.

29. Nagai N, Sakane N, Ueno LM, Hamada T, Moritan T. The -3826 A-->G variant of the uncoupling protein-1 gene diminishes postprandial thermogenesis after a high fat meal in healthy boys. J Clin Endocrinol Metab. 2003;88:5661-7.

30. Forga L, Corbalan M, Marti A, Fuentes C, MartínezGonzález MA, Martínez A. Influencia del polimorfismo -3826 A $Æ G$ en el gen de la UCP1 sobre los componentes del síndrome metabólico. An Sist Sanit Navar. 2003;26:231-6.

31. Heilbronn LK, Kind KL, Pancewicz E, Morris AM, Noakes M, Clifton PM. Association of -3826 G variant in uncoupling protein-1 with increased BMI in overweight Australian women. Diabetologia. 2000;43:242-4.

32. Kim KS, Cho DY, Kim YJ, Choi SM, Kim JY, Shin $\mathrm{SU}$, et al. The finding of new genetic polymorphism of 
UCP-1 A-1766G and its effects on body fat accumulation. Biochim Biophys Acta. 2005;1741:149-55.

33. Salopuro T, Lindstrom J, Eriksson JG, Valle TT, Hamalainen $\mathrm{H}$, llanne-Parikka $\mathrm{P}$, et al. Common variants in beta2- and beta3-adrenergic receptor genes and uncoupling protein 1 as predictors of the risk for type 2 diabetes and body weight changes. The Finnish Diabetes Prevention Study. Clin Genet. 2004;66:365-7.

34. Esterbauer H, Oberkofler H, Liu YM, Breban D, Hell $\mathbf{E}$, Krempler $\mathbf{F}$, et al. Uncoupling protein-1 mRNA expression in obese human subjects: the role of sequence variations at the uncoupling protein-1 gene locus. J Lipid Res. 1998;39:834-44.

35. Nakazaki M, Kakei M, Ishihara $H$, Koriyama $N$, Hashiguchi H, Aso K, et al. Association of upregulated activity of K(ATP) channels with impaired insulin secretion in UCP1-expressing insulinoma cells. J Physiol. 2002;540:781-9.

36. Bernal-Mizrachi C, Weng S, Li B, Nolte LA, Feng C, Coleman $\mathrm{T}$, et al. Respiratory uncoupling lowers blood pressure through a leptin-dependent mechanism in genetically obese mice. Arterioscler Thromb Vasc Biol. 2002;22:961-8.

37. Carroll AM, Porter RK. Starvation-sensitive UCP 3 protein expression in thymus and spleen mitochondria. Biochim Biophys Acta. 2004;1700:145-50.

38. Lengacher S, Magistretti PJ, Pellerin L. Quantitative rt-PCR analysis of uncoupling protein isoforms in mouse brain cortex: methodological optimization and comparison of expression with brown adipose tissue and skeletal muscle. J Cereb Blood Flow Metab. 2004;24:780-8.

39. Moulin K, Arnaud E, Nibbelink M, Viguerie-Bascands $\mathrm{N}$, Penicaud L, Casteilla L. Cloning of BUG demonstrates the existence of a brown preadipocyte distinct from a white one. Int J Obes Relat Metab Disord. 2001;25:1431-41.

40. Villarroya F, Iglesias R, Giralt M. PPARs in the control of uncoupling proteins gene expression. PPAR Res. 2007;2007:74364.

41. Meirhaeghe A, Amouyel P, Helbecque N, Cottel D, Otabe S, Froguel P, et al. An uncoupling protein 3 gene polymorphism associated with a lower risk of developing type II diabetes and with atherogenic lipid profile in a French cohort. Diabetologia. 2000;43:1424-8.

42. Damcott CM, Feingold E, Moffett SP, Barmada MM, Marshall JA, Hamman RF, et al. Genetic variation in uncoupling protein 3 is associated with dietary intake and body composition in females. Metabolism. 2004;53:458-64.

43. Schrauwen P, Xia J, Walder K, Snitker S, Ravussin E. A novel polymorphism in the proximal UCP3 promoter region: effect on skeletal muscle UCP3 mRNA expression and obesity in male non-diabetic Pima Indians. Int J Obes Relat Metab Disord. 1999;23:1242-5.

44. Krook A, Digby J, O'Rahilly S, Zierath JR, WallbergHenriksson $\mathrm{H}$. Uncoupling protein 3 is reduced in skeletal muscle of NIDDM patients. Diabetes. 1998;47:1528-31.

45. Clapham JC, Arch JR, Chapman H, Haynes A, Lister C, Moore GB, et al. Mice overexpressing human uncoupling protein-3 in skeletal muscle are hyperphagic and lean. Nature. 2000;406:415-8.

46. Choi CS, Fillmore JJ, Kim JK, Liu ZX, Kim S, Collier EF, et al. Overexpression of uncoupling protein 3 in skeletal muscle protects against fat-induced insulin resistance. J Clin Invest. 2007;117:1995-2003.

47. Esterbauer H, Schneitler C, Oberkofler H, Ebenbichler C, Paulweber B, Sandhofer F, et al. A common polymorphism in the promoter of UCP2 is associated with decreased risk of obesity in middle-aged humans. Nat Genet. 2001;28:178-83.

48. Ochoa MC, Santos JL, Azcona C, Moreno-Aliaga MJ, Martínez-González MA, Martínez JA, et al. Association between obesity and insulin resistance with UCP2-UCP3 gene variants in Spanish children and adolescents. Mol Genet Metab. 2007;92:351-8.

49. Aschner P, King H, Triana de Torrado M, Rodríguez BM. Glucose intolerance in Colombia. A populationbased survey in an urban community. Diabetes Care. 1993;16:90-3.

50. Mejía S, Vélez A, Buriticá O, Arango M, Del-Río J. La política farmacéutica nacional en Colombia y la reforma de la seguridad social: acceso y uso racional de medicamentos. Cad Saude Publica. 2002;18:1025-39.

51. Qi L, Hu FB, Hu G. Genes, environment, and interactions in prevention of type 2 diabetes: a focus on physical activity and lifestyle changes. Curr Mol Med. 2008;8:51932.

52. Colditz GA, Willett WC, Rotnitzky A, Manson JE. Weight gain as a risk factor for clinical diabetes mellitus in women. Ann Intern Med. 1995;122:481-6.

53. Leahy JL. Pathogenesis of type 2 diabetes mellitus. Arch Med Res. 2005;36:197-209.

54. Rothman KJ, Greenland S. Modern Epidemiology. Second edition. Filadelfia, EU: Lippincott-Raven; 1998.

55. Cheurfa N, Dubois-Laforgue D, Ferrarezi DA, Reis AF, Brenner GM, Bouche C, et al. The common $-866 \mathrm{G}>\mathrm{A}$ variant in the promoter of UCP2 is associated with decreased risk of coronary artery disease in type 2 diabetic men. Diabetes. 2008;57:1063-8. 